\title{
Interview with Louis Nirenberg
}

\author{
Martin Raussen and Christian Skau
}

Louis Nirenberg is one of the recipients of the 2015 Abel Prize of the Norwegian Academy of Science and Letters; the other is John Nash. The following interview with Nirenberg was conducted by Martin Raussen and Christian Skau in Oslo on May 18, 2015, in conjunction with the Abel Prize celebration. This article originally appeared in the December 2015 issue of the Newsletter of the European Mathematical Society www.ems-ph.org/journals/ news letter/pdf/2015-12-98.pdf, pp. 33-38, and is reprinted here with permission of the EMS. (An analogous interview with John Nash will appear in a future issue of the Notices.)

\section{Partial Differential Equations (and Geometry/ Physics)}

Raussen and Skau: Firstly, we want to congratulate you on being awarded (with Professor Nash) the Abel Prize for 2015. You will receive the Abel Prize from His Majesty the King of Norway in a ceremony tomorrow.

Your first important achievement in mathematics was solving the so-called Weyl problem in your PhD thesis. Could you tell us what the Weyl problem is about?

Professor Nirenberg: The problem was originally stated by Hermann Weyl. You have a two-dimensional sphere with a metric (that is, a way of measuring distance), and connected with the metric is its curvature. If this curvature is positive, the question is whether you can find a convex body in three-dimensional space with a mapping to the sphere so that when you measure the distance in Euclidean space, it agrees with the metric? Weyl went quite far toward solving this problem but there were some estimates missing. My contribution was to fill in those missing estimates.

When you express the problem mathematically, it involves partial differential equations. The equations were so-called non-linear partial differential equations and the problem was proving the existence of solutions of these equations. Much of my career has really been devoted to studying partial differential equations in general but also applying them to problems from geometry and complex analysis. I even wrote two papers with a friend in

Martin Raussen is professor of mathematics at Aalborg University, Denmark. His email address is raussen@math . aau.dk.

Christian Skau is professor of mathematics at the Norwegian University of Science and Technology, Trondheim, Norway. His email address iscsk@math.ntnu.no.

For permission to reprint this article, please contact: reprintpermission@ams.org.

DOI: http://dx.doi.org/10.1090/noti1330

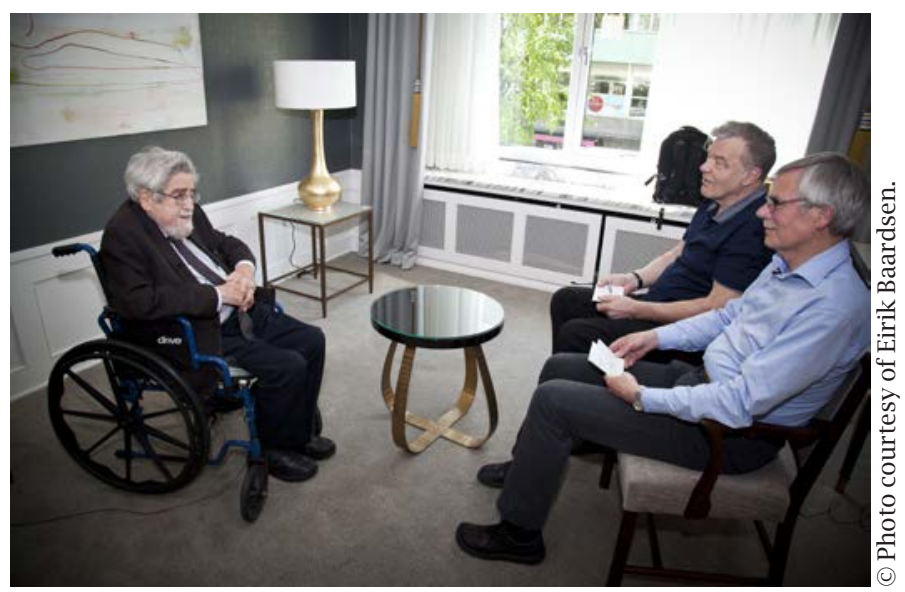

From left to right: Louis Nirenberg, Christian Skau, Martin Raussen.

economics involving partial differential equations. In my mind, it is a wonderful field. A big part of the problem is proving that solutions exist because equations can be written down for which one knows there are no solutions.

Raussen and Skau: Many of these problems come from physics so solutions would be expected to exist?

Professor Nirenberg: Yes. But, for instance, for equations in fluid dynamics (the so-called Navier-Stokes equations that were introduced one-hundred fifty years ago), mathematicians have not been able to prove that smooth solutions exist for all time. So that is still an open problem.

Raussen and Skau: Is it true that the best result in that direction is your joint work with Caffarelli and Kohn from 1982 ?

Professor Nirenberg: That result is not about the existence of solutions but about the dimension of singularities if they do occur. They cannot have a high dimension; for instance, they cannot fill a curve. They must fill a set of dimension less than one. You may wonder what the hell that is? It either has dimension zero or it has dimension 
one. But, no! There are concepts of dimension of any nonnegative number. We proved that the one-dimensional measure had to be zero, so the set could not have dimension one. The paper is very technical.

Raussen and Skau: But it is very important in connection with the Navier-Stokes equations?

Professor Nirenberg: Well, it is a useful result mathematically whether engineers use it or not. Aeroplanes fly whether we solve the Navier-Stokes equations or not. But it is a big mathematical challenge to show that there are smooth solutions.

Raussen and Skau: Do you often think about the NavierStokes equations?

Professor Nirenberg: Once in a while. But I don't really have any fresh ideas. I think it is up to younger people.

\section{Start of a Career in Mathematics}

Raussen and Skau: May we ask how your mathematical life started? We were told that a certain teacher of Hebrew played an instrumental role. Is that true?

Professor Nirenberg: My father tried to teach me Hebrew. I resisted, stupidly, and now I know no Hebrew at all. He hired a friend to give me lessons in Hebrew. This friend happened to like mathematical puzzles and half the lessons then consisted of these puzzles. I found them quite fascinating but, I must say now, at my age, I am no longer fascinated by puzzles. They are for young people.

That started my interest in mathematics. I also went to a very good high school. This was during the depression and to be a high school teacher was considered a very good job. I had excellent teachers and I must say that the quality of the students was also very good. I particularly enjoyed the mathematics courses and especially geometry and physics. I then decided I would like to study physics.

Raussen and Skau: Were there already clear signs that you had an exceptional talent for mathematics?

Professor Nirenberg: The teachers considered me good but I think it became clearer in college that I had some talent in mathematics. When I graduated from university, I actually received a gold medal for my work in mathematics and physics.

Raussen and Skau: You graduated from McGill University in Montreal. Perhaps you could tell us about your experience studying mathematics and physics at university?

Professor Nirenberg: I finished high school and applied for a scholarship at McGill, which I didn't get. The high school offered an additional year, equivalent to a first year at college. I did that, applied again to McGill and then got a scholarship. So I was at McGill for three years rather than the usual four. This was during World War II and I graduated in the spring of 1945, just at the end of the war in Europe. It was a pleasure to study mathematics and physics. However, that was the only thing I studied. Because I missed the first year, I didn't take any courses in other interesting subjects. I am sorry I didn't.

Raussen and Skau: How did you end up at the Courant Institute in New York?

Professor Nirenberg: By pure luck! When I finished at McGill, I had a summer job at the National Research Council where they did atomic research. A son of
Courant had married a young woman from Montreal, whom I knew. They both worked there and one day she said they were going to New York to visit Courant [1888-1972]. I asked her to ask him to suggest some place I could apply to do graduate work in physics. She came back and said that Courant suggested that I come and take a Master's degree in mathematics. I could then go on to do physics, he said. I went down for an interview and was offered an assistantship in mathematics. I got a Master's degree and I just stayed on. I never left New York University.

\section{Courant, Friedrichs and the CUNY}

Courant was head of a very famous institute in Göttingen, Germany. He was kicked out when the Nazis came to power but he was offered a position at New York University a year later to set up a graduate programme in the mathematics department. They only had undergraduate training at that time. He came to New York to do that but there were very few students in those first years. The number of students only increased after the war. When I came, just after the war, there were a number of very talented students. Some of them became well known mathematicians. I was part of a very good body of students and it was an exciting time.

Usually, when you get a PhD at some university in America, you then leave. You go to another university for your first job. Courant was different. He kept the good people. If good people got PhDs, he simply offered them jobs.

Raussen and Skau: Did it help being offered a job if you played an instrument?

Professor Nirenberg: I didn't play an instrument. But if I had, it may have helped even more. Of course, the rumor was that he hired people who played instruments (unless they played the piano, which he played himself).

Raussen and Skau: Did you meet with him often?

Professor Nirenberg: Oh, yes. He often invited the students socially to his home. His wife was completely devoted to music and played a number of instruments. She was the daughter of the mathematician Carl Runge [1856-1927], by the way. They had two daughters who were both very ardent musicians. One of them became a professional musician and is now married to Peter Lax. Courant was wonderful with young people-very encouraging and really exceptional.

Raussen and Skau: Mathematically speaking, your mentor was Kurt Friedrichs?

Professor Nirenberg: Yes. Friedrichs [1901-1982] was the person I regard as my Sensei (as the Japanese say). I really was most influenced by him. He worked mainly with partial differential equations but he also did other things. He wrote a book on quantum theory and a book, together with Courant, on shock wave theory, which was widely used and translated into many languages.

Raussen and Skau: You mentioned that there was a special atmosphere at the Courant Institute, in part because no distinction was made between pure and applied mathematics...

Professor Nirenberg: That's right. Courant insisted there was no difference between pure and applied mathematics. He did both and he encouraged people to do the same. It is just mathematics. When New York University 
hired him, they asked him what a mathematics department needs and he said: "A library and a coffee room." So we have a very nice lounge that we use all the time.

Raussen and Skau: It is remarkable that you are the fourth Abel Prize Laureate associated with the Courant Institute (after Peter Lax in 2005, Srinivasa Varadhan in 2007 and Mikhail Gromov in 2009). What has made this institute so successful?

Professor Nirenberg: Well, partly it is just the warm atmosphere. I think graduate students are very happy there and there is a lot of interaction between the students and the faculty. It is, of course, much bigger now than it was when I was a student there. But the warm atmosphere has prevailed.

Raussen and Skau: Who were your most important colleagues over your career?

Professor Nirenberg: There is Friedrichs but also two other students of Courant: Fritz John [1910-1994], a wonderfully talented mathematician who later became a faculty member (I had the fortune of writing one paper with him) and Hans Lewy [1904-1988] (I wrote several papers related to some of his work). Hans left Germany immediately after Hitler came to power. He came to the United States and had a career at Berkeley.

\section{Partial Differential Equations and Inequalities}

Raussen and Skau: Your name, often with various coauthors, is attached to many fundamental concepts and theorems in PDEs. If you just look at the citation list, your work has had a tremendous impact. Let's start with Fritz John, with whom you authored a very influential paper about BMO functions (BMO standing for "Bounded Mean Oscillation").

Professor Nirenberg: That was his idea. He introduced BMO functions. It came from some work he had done in elasticity theory. He approached me saying: "I have a class of functions and I believe they should have such-and-such a property." I worked on it and was able to prove that property. He then improved it so the final version is better than what I had done. It became a joint paper and I must say a lot of people have referred to it.

Raussen and Skau: Absolutely! It became famous-if we may say so-because of the many applications. For instance, Charles Fefferman got the Fields Medal in 1978 and one of his main contributions was to show that the BMO space is dual to the Hardy space H1.

Professor Nirenberg: Charles Fefferman did many things but in particular, he proved the duality result that you refer to.

Raussen and Skau: Your paper with Fritz John contains the John-Nirenberg inequality. You love inequalities?

Professor Nirenberg: I love inequalities. And what we proved in the paper was really an inequality.

Raussen and Skau: Would you explain why inequalities are so important in the theory of PDEs?
Professor Nirenberg: When you look at a partial differential equation, you may ask whether a solution exists. Now, you can't write down the solution so you need to know some bounds. It cannot be too big, it cannot be too negative, its derivatives cannot be too big and so on. You try to get estimates of the size of the function and of its derivatives. All these estimates are inequalities. You are not saying that something is equal to something but that something is less than some constant. Thus, inequalities play an essential role in proving the existence of solutions. In addition, you want to prove properties of solutions and, again, inequalities play a central role. Hence, inequalities are absolutely fundamental to studying partial differential equations; for that matter, so are they for the study of ordinary differential equations.

Raussen and Skau: Let's move on to your joint research with Shmuel Agmon and Avron Douglis [1918-1995]. There were two very important papers. Can you explain what they contained?

Professor Nirenberg: What we did was to extend some classic work, by the Polish mathematician Schauder [18991943], to higher order equations. There is a fundamental paper of Schauder for second-order, so-called elliptic equations. We thought it would be useful for people to be able to deal with higher order equations and systems of equations so we proved the analogues of those results. In the other paper, we proved the results for systems and also for different norms, that is, for different ways of measuring the size of the solutions. We published several different kinds of inequalities and they have been used by many people.

Raussen and Skau: You wrote a paper with Joseph Kohn introducing the important notion of pseudo-differential operators. You are one of the fathers of that concept. Can you explain why this concept is so important and how you came upon it?

Professor Nirenberg: Joe Kohn had published a fundamental paper in complex analysis. It involved the regularity of solutions for a certain class of systems up to the boundary - a rather difficult paper! He suggested we should try to generalize this to more general systems of equations. We started to look at it and we had to consider so-called commutators of operators. You apply an operator and then you apply a second one. Then you take the difference of that result with the operator obtained by applying the second one and then the first. We needed properties of the commutator. We were using a certain space, called an Lp-space, and a theory due to Calderón [1920-1998] and Zygmund [1900-1992] for certain singular integral operators. We needed to extend their result to commutators so we thought: "How do we extend these singular integral operators to make an algebra out of them?"

That led to what we call pseudo-differential operators. The concept came from a very specific problem in systems of partial differential equations but it turned out to be a useful thing in itself. It grew out of Calderón and Zygmund's theory. By the way, Calderón was a wonderful mathematician and he danced the tango, which I admired enormously. 


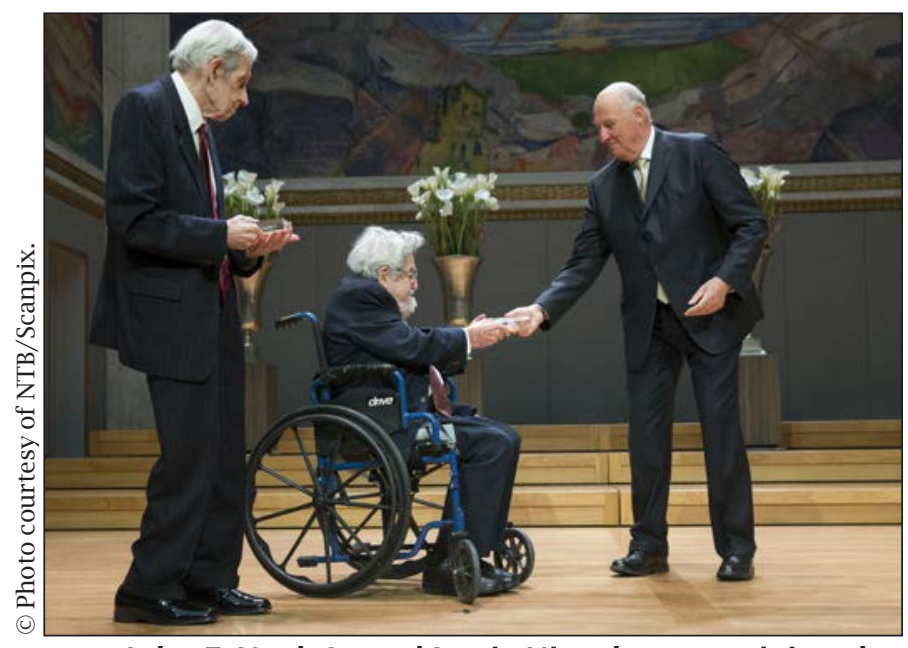

John F. Nash Jr. and Louis Nirenberg receiving the 2015 Abel Prize from His Majesty King Harald at the award ceremony in Oslo on May 19, 2015.

Raussen and Skau: You had a very bright student, August Newlander, with whom you wrote a very important joint paper in 1957. Can you tell us about the results you proved there?

Professor Nirenberg: It was a problem I first heard of from André Weil [1906-1998]. He said: "Here's a problem in complex analysis. Why don't you people in partial differential equations work on this kind of problem?" I thought: "Why not? Let's try." I took a student who was very bright and I said: "Let's look at the very simplest case, in the lowest dimension." The student, Newlander, had the initial idea, which worked fine in low dimensions but, to our surprise and dismay, didn't work in higher dimensions. We had to come up with a completely different proof in higher dimensions. It led from a linear problem to a nonlinear problem. It was kind of strange but the non-linear problem was in some ways more accessible.

Raussen and Skau: What was André Weil's reaction when you solved the problem?

Professor Nirenberg: He was very happy and so were other people in complex analysis. Many people have used the result. Some years later, Hörmander [1931-2012] found a linear proof of the same result. It was very technical but it was purely linear.

Raussen and Skau: Are there any outstanding problems in the enormous field of partial differential equations, apart from the Navier-Stokes problem, that you would like to highlight?

Professor Nirenberg: Well, I think almost nothing has been done in so-called over-determined systems, that is, where there are more equations than unknowns. You may have two unknowns and five equations so there have to be some compatibility relations. There's almost no analytic theory of that. There is a theory developed by Cartan [1869-1951] and Kähler [1906-2000] but that assumes that everything is analytic. Outside analytic category, almost nothing is known about such systems. They often come up in geometry so I feel that this is a big gap in the theory of partial differential equations.

\section{Mathematics and Mathematicians All Over the World}

Raussen and Skau: May we ask you some questions about international mathematics? We know that you travelled to post-war Europe very soon after your graduation.

Professor Nirenberg: Yes. I had a fellowship and came to Zurich during the academic year 1951-52. I went mainly to be with Heinz Hopf [1894-1971], who was a geometer and a topologist. Heinz Hopf was a wonderful person-a lovely and extremely kind man. I also spent one month in Göttingen that year. That was arranged by Courant who felt I should go there. During that year, I didn't actually carry out any research. What I did was to write up the things I had done before. I had been very slow at writing them up for publication because I somehow had a block against writing. So during that year I wrote several papers.

Raussen and Skau: Did Courant ever return to Göttingen?

Professor Nirenberg: Yes. After the war, he went back to Germany many times. He had many contacts and he wanted to help build up German mathematics again.

Raussen and Skau: He must also have been very bitter?

Professor Nirenberg: Well, he was bitter but, at the same time, he had friends and he wanted to encourage and help to develop mathematics in Germany.

Raussen and Skau: You also went to the Soviet Union?

Professor Nirenberg: Yes. The first time I went was in 1963. It was a joint Soviet-American symposium on partial differential equations, arranged by Courant on one side and the Soviet mathematician Lavrentyev [1900-1980] on the other. There were about two dozen American mathematicians and about one hundred twenty Soviet mathematicians from all over the Soviet Union. It is one of the best meetings I have ever attended. It was in Novosibirsk, Siberia, which was the academic city that Lavrentyev had helped create. It was like being aboard a ship for two weeks with people you make friendships with immediately. I made friends with Russians that are still friends today. Some have died, unfortunately, but I have had very good friends in Russia since then. I have never collaborated with any of them but they are still very warm friends; we would meet and talk about mathematics, politics and all kind of things.

Raussen and Skau: How about China?

Professor Nirenberg: I have been to China a number of times. The first visit was arranged by Chern [1911-2004], a Chinese mathematician who had settled in America. This was in 1975 and the Cultural Revolution was still going on, though I didn't realize it at the time. For instance, I was visiting the Chinese Academy of Science but I was taken to Beijing University. I said I would like to meet the faculty but they said they were busy teaching-which was simply a lie. There was no teaching going on. They showed me the library and then they wanted to take me to some other university but I said: "There's no point. Either I meet the faculty or I don't go."

They had me give many lectures but I said I also wanted to hear what some of the people there were doing. So some young people spoke about some of their research. I learned later that they had to get permission to attend my 
lectures. I didn't make close friends at that time. It was an interesting experience and, of course, things have changed enormously since then. I did make friends with some who subsequently came and spent a year or two at Courant.

Raussen and Skau: We should also mention that you were awarded the first Chern Medal of the International Mathematical Union.

Professor Nirenberg: Yes. That's true. That was in 2010.

Raussen and Skau: You were also awarded the first Crafoord Prize in 1982, together with Arnol'd [1937-2010]. Perhaps it was a tongue-in-cheek comment but Arnol'd once said something like: "Mathematics is the part of physics in which experiments are cheap."

Professor Nirenberg: It wasn't entirely tongue-in-cheek. He really felt that the contact of mathematics with physics and the real world was important.

He didn't get permission to go and get the Crafoord Prize. I visited Moscow just before I went to Sweden and had dinner with him in his home. He was waiting until the last minute to see if he would get permission, but he didn't.

When I went back to America, I got a call from a woman claiming to be Arnol'd's sister. I thought: "How is that possible?" I had just seen Arnol'd a few weeks before and he never mentioned he had a sister in New Jersey. She came to my office and, indeed, it was Arnol'd's sister. He never mentioned a word. It's incredible!

Raussen and Skau: Talking about Arnol'd, on some occasions he expressed frustration that results proved in the West had already been proved in Russia but, because of poor communication during the Cold War, these results were not known. Did he express these feelings to you?

Professor Nirenberg: He tended to do that. I remember once he was visiting New York. Someone was giving a seminar talk and he was attending the lecture. During the talk, Arnol'd said: "Oh, that was already proved by suchand-such a Russian." But the person giving the seminar talk then checked and the Russian had never proved it. So Arnol'd was not always correct. He tended to give more credit to Russians than was due. You may have heard the joke where the Russian says: "What you proved, I proved first. And anyway, the result is trivial."

\section{Problems, Collaboration and "Sitzfleisch"}

Raussen and Skau: It is striking that 90\% of your published papers describe joint work. Can you explain why this is so?

Professor Nirenberg: It is just a pleasure! It is just an enormous pleasure talking mathematics with others and working with them. Of course, much of the work you do yourself. I mean, you discuss ideas and work with others but then you go home and think about what you have done. You get some ideas and you get together again and talk about the new ideas. You get reactions to your ideas and you react to their ideas. It is a wonderful experience.
Raussen and Skau: Do you usually start out with a goal in mind?

Professor Nirenberg: Usually there is a goal. But somebody once used the expression: "There are those mathematicians who, when they come to a fork in the road, they take it." I'm that kind of mathematician. So, I may be working on a problem with a colleague when we come to something that looks interesting, and we explore that and leave the original problem for a while.

Raussen and Skau: Are you more of a problem solver?

Professor Nirenberg: Yes, definitely. There are two kinds of mathematicians. There are those who develop theories and those who are primarily problem solvers. I am of the latter.

Raussen and Skau: Do you come up with interesting problems through discussions with other mathematicians? What kinds of problems are you attracted to? Is there any pattern?

Professor Nirenberg: It's hard to say. A graduate student once asked me how I find good research problems. I said to him that I sometimes see a result but don't like the proof. If the problem appeals to me, I start to think if there is a better proof. My ideas may lead to a better proof or may lead to something new. The student said he'd never seen a proof he didn't like and I thought: "He is hopeless!"

Raussen and Skau: May we ask you a question that we have asked several previous laureates? How does one find the proof of a mathematical result? Some people work with perseverance until a proof is complete but others tell us that insight appears in a sudden flash-like lightning. Do you have experiences of this sort?

Professor Nirenberg: Both may happen. But most of the time you are stuck. Maybe you make a breakthrough with some problem as you get some insight and see something you didn't see before.

But the perseverance and all the work you carried out before seems to be necessary to have this insight. You need perseverance or, as the Germans say, you need "Sitzfleisch".

Raussen and Skau: Are you the kind of person that gets so involved in trying to solve a problem that you are, so to speak, lost to the world?

Professor Nirenberg: Not all the time but it can happen for many hours. Sometimes, I wake in the middle of the night and start thinking about a problem for hours and cannot sleep. When you do that, it is very hard to fall asleep again! If I have an idea, I just follow it. I see if it leads to something. I still try to do that but in the last few years it has not led anywhere. I haven't had any success.

\section{Communicating Mathematics}

Raussen and Skau: You have had forty-five PhD students. That is an impressive number! Can you tell us what your philosophy is? How do you come up with problems for your students?

Professor Nirenberg: It's hard to say. Sometimes it is hard to think of a suitable problem. It is easier to think 
of problems that are too hard, and just not practical, than to think of a problem that is good and can be solved in reasonable time. I can't really answer that question. I don't know how I go about posing problems.

Raussen and Skau: Were there occasions when you had to help students along?

Professor Nirenberg: Oh, yes. I meet the students regularly, usually once a week. We discuss their progress and I might make suggestions. I may say: "Look at this paper, this may lead to something."

Raussen and Skau: How would you describe your love for mathematics? What is it about mathematics that is so appealing to you? Is it possible to communicate this love to people outside the mathematical community? Does one have to be a mathematician to appreciate the appeal of mathematics?

Professor Nirenberg: Some people are very good at communicating to the general public. I am not so very good at that. But once you are in it, once you are hooked, it's very exciting and fun. I have used the word "fun" before. But it is really fun to do mathematics. It is an enormous pleasure to think about mathematics even though you are stuck 90 percent of the time, perhaps even more.

Raussen and Skau: That is what people outside mathematics cannot comprehend.

Professor Nirenberg: Yes, it is hard to comprehend. You have to be in it and I think it does take some talent to be able to do mathematics. But it also takes, as I said, "Sitzfleisch." You need to be stubborn and have perseverance, and you can't give up. I have been stuck on some problems for years.

Raussen and Skau: But you do think it's important to try to communicate to the general audience?

Professor Nirenberg: Yes, I do think that is important: (a) for the development of mathematics, and (b) to show them that it is a pleasure to do mathematics. Courant and Robbins [1915-2001] wrote a very nice book: 'What is Mathematics?'. It is a lovely book. There is also a recent book by Edward Frenkel, a mathematician who came as an immigrant from Russia as a young man. It is called 'Love and Math'. He makes a valiant attempt to get the general public interested in the branch of mathematics in which he works (which is also connected to physics). It is very hard to do. He tries but I think it is too hard for the general public. But he makes a real attempt to do it and I must say I admire him for that. I just recently read his book.

\section{Music and Movies}

Raussen and Skau: We have one final question that we have asked several laureates before. What are you interested in when you are not doing mathematics?

Professor Nirenberg: I love music. I love movies. You won't believe this but at the time when I lived in Montreal, in the province of Québec, you could not get into a movie before you were sixteen. Incredible! Now it's hard to believe. So when I was sixteen, I went crazy and started to go to movies. When I moved to New York, there were suddenly all these foreign movies: Italian movies, Russian movies, French movies. I went crazy. I went almost every night to the movies. Since then, I have loved movies.
Raussen and Skau: Have you seen "A Beautiful Mind"? Professor Nirenberg: Of course, and I have read the book.

Raussen and Skau: What kind of music do you like?

Professor Nirenberg: Mainly classical but I also listen to jazz. My grandson, who will be at the ceremony tomorrow, is a professional jazz drummer. And I love Argentinian tango. I have a large collection of records of Argentinian tango.

Raussen and Skau: Not only on behalf of us but also on behalf of the Norwegian, Danish and European Mathematical Societies, we would like to thank you for a very interesting interview. 